\title{
Application of headspace solid-phase microextraction and gas chromatography for the analysis of furfural in crude palm oil.
}

\begin{abstract}
Processing of vegetative material containing pentoses has been shown to result in the formation of furfural. Furfural exhibits a spectrophotometric absorption peak at $518 \mathrm{~nm}$ when complexed with aniline acetate. Headspace solid-phase microextraction (HS-SPME) method has been successfully used to confirm the presence of furfural in crude palm oil (CPO). Solid phase microextraction (SPME) fiber composed of divinylbenzene/Carboxen/polydimethylsiloxane (DVB/PDMS/CAR) was used to absorb the volatiles in the headspace of the oil. The isolated compounds from the fiber was desorbed and separated on a capillary polar column of a gas chromatograph. Response surface methodology (RSM) was used to optimize the SPME fiber condition for maximum absorption of furfural from CPO. The optimized temperature and time for furfural extraction onto the SPME fiber are $70{ }^{\circ} \mathrm{C}$ for $40 \mathrm{~min}$. Oils obtained from the mill were found to contain between 2 and $13 \%$ furfural.
\end{abstract}

Keyword: Furfural; Sterilization; Hemicelluloses; SPME. 